\title{
Direct Axisymmetric Vlasov Simulations of Space Charge Dominated Beams
}

\author{
F. Filbet ${ }^{1}$, J.-L. Lemaire ${ }^{2}$, E. Sonnendrücker ${ }^{3}$ \\ 1 IRMA, Université Louis Pasteur, F-67084 Strasbourg Cedex, \\ filbet@math.u-strasbg.fr \\ 2 CEA, B.P.12, F-91680 Bruyères-le-Châtel, jlemaire@bruyeres.cea.fr \\ 3 IRMA, Université Louis Pasteur, F-67084 Strasbourg Cedex, \\ sonnen@math.u-strasbg.fr
}

\begin{abstract}
A numerical method for the direct simulation of the axisymmetric Vlasov equation is introduced. It is based on a modified formulation of the Vlasov equation using the invariance of the canonical angular momentum. This leads in particular to a straightforward and very efficient parallel algorithm. Then it is applied to simulations of a RMSmatched semi-Gaussian beam and a perturbed thermal equilibrium.
\end{abstract}

\section{Introduction}

Eulerian direct Vlasov simulation of space charge dominated beams has proven to be an efficient alternative to PIC methods as it is completely devoid of numerical noise. It enables in particular to get a better insight into phenomena happening at the edge of the beam where the distribution function is very small. These regions are generally described by too few particles in PIC simulations.

We shall describe a three-dimensional $r, v_{r}, v_{\theta}$ axisymmetric transverse solver involving fewer dimensions than a comparable cartesian solver, which would be four dimensional in phase space, enabling us to use a larger number of grid points.

The method is based on the use of the canonical angular momentum which is invariant, and thus only appears as a parameter in the equations. Thanks to the use of this invariant, a straightforward very efficient parallelization is achieved.

The code is then validated on two test-cases involving heavy ions, the evolution of a transverse space-charge wave in a RMS-matched semi-Gaussian beam and the formation of a halo in a beam where a perturbation from a MaxwellBoltzmann thermal equilibrium is introduced.

The outline of the paper is as follows : We shall first recall the axisymmetric Vlasov equation and its properties. Then, we present the discretization of the axisymmetric Vlasov equation. And finally we present numerical results for the cases of a semi-Gaussian beam and a perturbed thermal equilibrium. 


\section{The axisymmetric Vlasov equation}

We consider an axisymmetric beam uniform in the longitudinal direction. It can be represented by the axisymmetric Vlasov equation, which describes the evolution of a species of charged particles under applied and self-consistent fields, and reads

$$
\frac{\partial f}{\partial t}+v_{r} \frac{\partial f}{\partial r}+\left(\frac{q}{m}\left(E_{s}+E_{a}\right)+\frac{q B_{z}}{m} v_{\theta}+\frac{v_{\theta}^{2}}{r}\right) \frac{\partial f}{\partial v_{r}}-\left(\frac{q B_{z}}{m} v_{r}+\frac{v_{\theta} v_{r}}{r}\right) \frac{\partial f}{\partial v_{\theta}}=0 .
$$

where the distribution function $f$ is a function of radial position $r$, velocity $\left(v_{r}, v_{\theta}\right)$ and time $t$. We assume here that the applied magnetic field is longitudinal and uniform, i.e. $B=\left(0,0, B_{z}\right)$, where $B_{z}(t)$ only depends on time. The associated vector potential then has only a non vanishing $A_{\theta}$ component the value of which is $A_{\theta}=\frac{r}{2} B_{z}$. The self-consistent electric field $E_{s}(t, r)$, deriving from a scalar potential $\phi_{s}$ is given by the axisymmetric Poisson equation which reads

$$
\frac{1}{r} \frac{\partial r E_{s}}{\partial r}=\rho(t, r) / \varepsilon_{0}, \quad \rho(t, r)=q \int_{\mathbb{R}^{2}} f\left(t, r, v_{r}, v_{\theta}\right) d v_{r} d v_{\theta} .
$$

The characteristic curves of the axisymmetric Vlasov equation are the solutions of the following differential system

$$
\left\{\begin{aligned}
\dot{r} & =v_{r} \\
\dot{v}_{r} & =\frac{v_{\theta}^{2}}{r_{2}}+\frac{q}{m} v_{\theta} B_{z}+\frac{q}{m} E_{s} \\
\dot{v}_{\theta} & =-\frac{v_{\theta}}{r}-\frac{q}{m} v_{r} B_{z} .
\end{aligned}\right.
$$

Classical invariants of the axisymmetric Vlasov equation (1) are the Hamiltonian

$$
H\left(r, v_{r}, v_{\theta}\right)=\frac{1}{2} m v_{r}^{2}+\frac{1}{2 m}\left(m v_{\theta}-q \frac{r}{2} B_{z}\right)^{2}+q \phi_{s}
$$

and the canonical angular momentum

$$
P\left(r, v_{\theta}\right)=m r v_{\theta}+\frac{r^{2}}{2} q B_{z}
$$

Let us make use of this last invariant, as suggested in [3], to simplify equation (1). Denoting by $I=\frac{P}{m}$ and making the change of variable $\left(r, v_{r}, v_{\theta}\right) \rightarrow\left(r, v_{r}, I\right)$ with

$$
v_{\theta}=\frac{I}{r}-\frac{1}{2} \frac{q B_{z}}{m} r
$$

we get

$$
\frac{\partial f}{\partial t}+v_{r} \frac{\partial f}{\partial r}+\left(\frac{q}{m} E_{s}(t, r)+\frac{I^{2}}{r^{3}}-\frac{1}{4}\left(\frac{q B_{z}}{m}\right)^{2} r\right) \frac{\partial f}{\partial v_{r}}=0, \quad \forall I \in \mathbb{R} .
$$

This new formulation of the axisymmetric Vlasov equation is particularly well adapted to parallelization as the variable $I$ only plays the role of a parameter. 


\section{Discretization of the axisymmetric Vlasov equation}

We use a grid in phase space $\left(r, v_{r}, I\right)$. Yet it is necessary to take particular care of the $I$ direction. Indeed, when the self consistent electric field is linear, as for the $\mathrm{K}-\mathrm{V}$ distribution function, the characteristic curves associated to Eq. (6), along which the distribution function is constant, are of the form

$$
\frac{\omega^{2}}{2} r^{2}+v_{r}^{2}+\frac{I^{2}}{r^{2}}=\text { const. }
$$

Hence it is necessary to control the ratio $I / r$, therefore we discretize the $I$ direction so that

$$
I= \pm \omega r^{2} \text {. }
$$

We then use the conservation of the distribution function along the characteristics to devise the numerical algorithm which will be based on the semiLagrangian methodology [5]. The new values are computed at the grid points in two steps: (i) compute the origin of the characteristic ending at the grid point one time step back, (ii) interpolate the value of the distribution function there, which is also the new value at the grid point, from the old values at the surrounding grid points. This method is not subject to a Courant condition on the time step which would be very restrictive near the axis $r=0$.

In the axisymmetric Vlasov equation the $I^{2} / r^{3}$ factor acts like a repulsion potential with respect to the total electric field. This potential is largest near the axis, where the electric field is negligible. As usual for axisymmetric problems, the major difficulty in the discretization of the Vlasov equation in cylindrical coordinates lies in the handling of the equation near the axis $r=0$. The most natural method would consist in separating the free transport part which can be solved explicitly from the self-consistent part. However, numerical errors would be generated near the axis and propagate inside the domain, and our goal here is to devise a very precise numerical method for which this is unacceptable. So, we shall go with a classical operator splitting method, and split between advection in $r$ and advection in $v_{r}$.

On the time interval $\left[t^{n}, t^{n+1}\right]$ we proceed as follows: the distribution function at time $t^{n}$ is given by $f^{n}\left(r, v_{r}, I\right)$, we first compute $f^{*}$ such that

$$
\left\{\begin{array}{l}
\frac{\partial f^{*}}{\partial t}+v_{r} \frac{\partial f^{*}}{\partial r}=0, \\
f^{*}\left(0, r, v_{r}, I\right)=f^{n}\left(r, v_{r}, I\right) .
\end{array}\right.
$$

We then compute the self-consistent electric field $E_{s}$ from the intermediate approximation $f^{*}\left(\Delta t, r, v_{r}, I\right)$. Then $f^{* *}$ such that,

$$
\left\{\begin{array}{l}
\frac{\partial f^{* *}}{\partial t}+\left(\frac{q}{m} E_{s}(t, r)+\frac{I^{2}}{r^{3}}-\frac{1}{4}\left(\frac{q B_{z}}{m}\right)^{2} r\right) \frac{\partial f^{* *}}{\partial v_{r}}=0, \\
f^{* *}\left(0, r, v_{r}, I\right)=f^{*}\left(\Delta t, r, v_{r}, I\right) .
\end{array}\right.
$$


Finally, $f^{n+1}\left(r, v_{r}, I\right)=f^{* *}\left(\Delta t, r, v_{r}, I\right)$.

The discretization of equation (7) requires to apply artificial boundary conditions. Actually, for this equation, at $r=0$ and for $v_{r}>0$, the particle flux is incoming, whereas particles with velocity $v_{r}<0$ leave the computational domain. Thus, we need to model how the particles cross the axis $r=0$. This can be done by imposing specular reflection conditions:

$$
f\left(0, v_{r}, I\right)=f\left(0,-v_{r}, I\right), \quad \forall v_{r}>0 .
$$

The numerical resolution of transport equations (7) and (8) is then performed using a semi-Lagrangian method with a cubic Hermite interpolation, using the values of the function and its derivative at the end points of the interval. Let us describe it in details for equation (7). On an interval $\left[r_{i}, r_{i+1}\right]$, we approximate the derivative of the distribution function at each grid point by a fourth order finite difference scheme:

$$
\partial_{r} f_{i}^{n}=\frac{1}{12 \Delta r}\left[8\left[f_{i+1}^{n}-f_{i-1}^{n}\right]-\left[f_{i+2}^{n}-f_{i-2}^{n}\right]\right] .
$$

The polynomial reconstruction is then given on each interval $\left[r_{i}, r_{i+1}\right]$ by the cubic polynomial interpolating the distribution function and its derivatives on the grid

$$
\begin{aligned}
f^{n}(r) & =f_{i}^{n}+\left(r-r_{i}\right) \partial_{r} f_{i}^{n}+\left(r-r_{i}\right)^{2}\left[3\left[f_{i+1}^{n}-f_{i}^{n}\right]-\Delta r\left[2 \partial_{x} f_{i}^{n}+\partial_{x} f_{i+1}^{n}\right]\right] \\
& +\left(r-r_{i}\right)^{3}\left[\Delta x\left[\partial_{r} f_{i+1}^{n}+\partial_{x} f_{i}^{n}\right]-2\left[f_{i+1}^{n}-f_{i}^{n}\right]\right] .
\end{aligned}
$$

This formula allows to evaluate the distribution function anywhere on the grid. It only remains to use the characteristic curves which can be solved explicitly on each split step to compute the distribution function at the grid points at time $t^{n+1}$.

\section{Numerical results}

\subsection{Semi-Gaussian beam}

We want to study here the evolution of an axisymmetric semi-Gaussian beam. Therefore we solve the Vlasov-Poisson in cylindrical coordinates, with an applied uniform and constant longitudinal magnetic field $B_{z}$. Then the distribution satisfies the Vlasov equation 6 . The initial distribution function describing a semi-Gaussian beam in is given by

$$
f_{0}\left(x, y, v_{x}, v_{y}\right)=\frac{n_{0}}{\left(2 \pi v_{t h}^{2}\right)\left(\pi a^{2}\right)} \exp \left(-\frac{\left(v_{x}-\frac{q B_{z}}{2 m} y\right)^{2}+\left(v_{y}+\frac{q B_{z}}{2 m} x\right)^{2}}{2 v_{t h}^{2}}\right),
$$

for $x^{2}+y^{2} \leq a^{2}$ and $f_{0}\left(x, y, v_{x}, v_{y}\right)=0$, if $x^{2}+y^{2}>a^{2}$. The magnetic field $B_{z}$ and the thermal velocity $v_{t h}$ are computed from RMS quantities, so that the beam is equivalent to a matched $\mathrm{K}-\mathrm{V}$ beam. 
The beam particles are singly ionized potassium $(Z=1, m=39.1$ amu $)$. The density $n_{0}$ is computed from the current $I=0.2 A$ and the beam velocity along the $z$-axis

$$
v_{z}=c \sqrt{\frac{\gamma^{2}-1}{\gamma^{2}}}, \quad \gamma=1+\frac{q}{m c^{2}} K,
$$

where $K$ is the beam kinetic energy $K=8.10^{4} \mathrm{eV}$. Finally the beam radius is $a=0.02 m$ and the tune depression is $\omega / \omega_{0}=1 / 4$.

We observe a space charge wave starting from the edge of the beam, propagating inwards and finally being reflected on the axis $r=0$. The initial selfconsistent field is linear within the beam. The variations are relatively weak but sufficient to strongly perturb the density (Fig. 1). Solving the axisymmetric equation allows us to eliminate one direction and thus to use a finer mesh than in the cartesian case and describe the distribution function more precisely. Moreover our new formulation of the Vlasov equation conserves the invariant $I=r v_{\theta}+\frac{q B_{z}}{2 m} r^{2}$.

This method gives very satisfying results for the present test case. The results are comparable to those obtained with a cartesian code, as presented in [6], for the same resolution and the code is much faster. Moreover it is possible to go to much finer resolutions and then diminish the numerical damping.

Table 1. Computational time for a $2 D \times 2 D$ cartesian and axisymmetric solvers.

\begin{tabular}{|c|c|c|}
\hline Number of processors & 2D Cartesian solver PFC & Axisymmetric Solver \\
\hline 4 processors & $178 \mathrm{~min}$ & $59 \mathrm{~min}$ \\
\hline 8 processors & $89 \mathrm{~min}$ & $27 \mathrm{~min}$ \\
\hline
\end{tabular}

\subsection{Perturbed thermal beam}

We start now from a dimensionless Maxwell-Boltzmann distribution $(q=m=1)$

$$
f_{0}\left(r, v_{r}, v_{\theta}\right)=\frac{\alpha}{2 \pi} \exp (-H)
$$

where $H$ is the dimensionless Hamiltonian obtained from (4), coupled with the Poisson equation

$$
-\frac{1}{r} \frac{\partial}{\partial r}\left(r \frac{\partial \phi_{s}}{\partial r}\right)=\alpha \exp \left(-\phi_{s}-\frac{r^{2}}{4}\right) .
$$

When $\alpha$ is different from zero or one, there are no analytical solutions of Poisson's equation (10). Therefore, we approximate the potential $\phi_{s}$ using a finite 

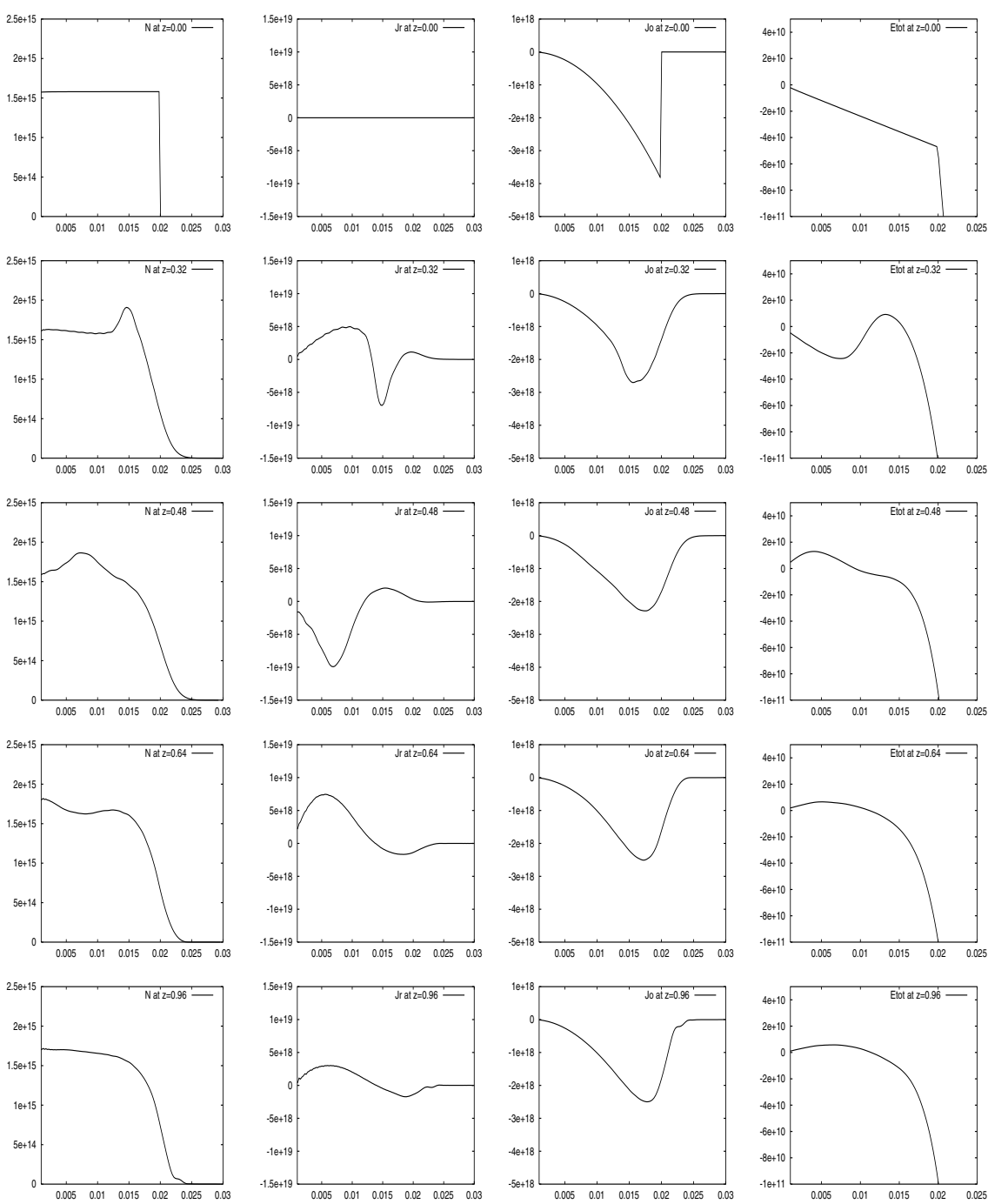

(1)

(2)

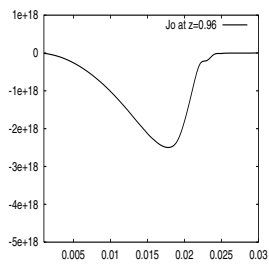

(3)

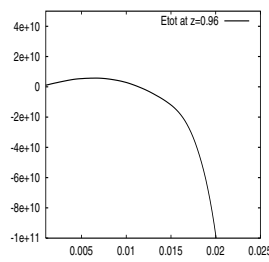

$(4)$

Fig. 1. Semi-Gaussian beam. Snapshots of slices of: (1) density $N(t, r)$, (2) first moment in $v_{r}$, (3) first moment in $v_{\theta}$, (4) total force field $E_{s}(t, r)-\left(\frac{q B_{z}}{2 m}\right)^{2} r$ inside the beam at times $z=0,0.32,0.48,0.64,0.96 \mathrm{~m}$. 
difference method : let $\left(r_{i}\right)_{i \geq 0}$ be a mesh of $\left.] 0, r_{\max }\right]$

$$
\begin{aligned}
\phi_{0} & =0 \\
\phi_{1} & =\alpha \Delta r^{2} / 4 \\
\phi_{i} & =\frac{r_{i-1}}{r_{i-1 / 2}}\left(2 \phi_{i-1}-\phi_{i-2}\left(\frac{r_{i-3 / 2}}{r_{i-1}}-\alpha \Delta r^{2} \exp \left(-\phi_{i-1}-r_{i-1}^{2} / 4\right)\right)\right) .
\end{aligned}
$$

The parameter $\alpha$ is then determined by the tune-depression. Thus, we obtain a steady-state solution of the self-consistent Vlasov-Poisson system. The density is then increased by $50 \%$. The beam parameters are the following : particles are singly ionized potassium $(Z=1, m=39.1 \mathrm{amu})$, current is $I=0.2 \mathrm{~A}$, energy $K$ $=8.10^{4} \mathrm{eV}$ and radius $r_{\max }=0.01 \mathrm{~m}$. We first display snapshots of the RMS quantities

$$
r_{r m s}=\left(\overline{r^{2}}\right)^{\frac{1}{2}}, \quad v_{r_{r m s}}=\left(\overline{v_{r}^{2}}\right)^{\frac{1}{2}}, \quad v_{\theta_{r m s}}=\left(\overline{v_{\theta}^{2}}\right)^{\frac{1}{2}},
$$

and the RMS emittance $\epsilon_{x}$, given by

$$
\epsilon_{x}=\left(\overline{x^{2} v_{x}^{2}}-{\overline{x v_{x}}}^{2}\right)^{\frac{1}{2}} .
$$

As, $x=r \cos \theta$

$$
\begin{aligned}
\overline{x^{2}} & =\int_{\mathbb{R}^{4}} x^{2} f\left(t, x, y, v_{x}, v_{y}\right) d x d y d v_{x} d v_{y} \\
& =\int_{\mathbb{R}^{3}} r^{2} f\left(r, v_{r}, I\right) d r d v_{r} d I\left(\int_{0}^{2 \pi}(\cos \theta)^{2} d \theta\right) \\
& =\pi \overline{r^{2}}
\end{aligned}
$$

Then, as $v_{x}=v_{r} \cos \theta-v_{\theta} \sin \theta$

$$
\begin{aligned}
\overline{v_{x}^{2}}= & \int_{\mathbb{R}^{4}} v_{x}^{2} f\left(t, x, y, v_{x}, v_{y}\right) d x d y d v_{x} d v_{y} \\
= & \int_{\mathbb{R}^{3}} v_{r}^{2} f\left(r, v_{r}, I\right) d r d v_{r} d I\left(\int_{0}^{2 \pi}(\cos \theta)^{2} d \theta\right) \\
& +\int_{\mathbb{R}^{3}} v_{\theta}^{2} f\left(r, v_{r}, I\right) d r d v_{r} d I\left(\int_{0}^{2 \pi}(\sin \theta)^{2} d \theta\right) \\
= & \pi\left(\overline{v_{r}^{2}}+\overline{v_{\theta}^{2}}\right) .
\end{aligned}
$$

Finally, $x v_{x}=r v_{r}(\cos \theta)^{2}-r v_{\theta}(\sin \theta)^{2}$,

$$
\begin{aligned}
\overline{x v_{x}} & =\int_{\mathbb{R}^{4}} x v_{x} f\left(t, x, y, v_{x}, v_{y}\right) d x d y d v_{x} d v_{y} \\
& =\pi \overline{r v_{r}} .
\end{aligned}
$$

which allows us to compute the emittance in cylindrical coordinates.

$$
\epsilon_{x}=\pi \sqrt{\overline{r^{2}}\left(\overline{v_{r}^{2}}+{\overline{v_{\theta}^{2}}}^{2}-{\overline{r v_{r}}}^{2}\right.} .
$$


Fig. 4 displays the evolution of the beam density, through slice plots on a logarithmic scale. It appears that, for a tune depression $\omega / \omega_{0}=1 / 2$, a plateau is formed at a density of around one thousandth of the core density. The snapshots are taken at times when the RMS value $v_{r_{r m s}}$ is at an extremum as it is there where the halo can be best observed [4]. Our beam has a radius of $0.01 \mathrm{~m}$ and the dimension of the plateau is approximatively of $0.025 \mathrm{~m}$, which corresponds to the maximal radius predicted by the empirical formula given in T.P. Wangler et al. [7] which is $0.0241 \mathrm{~m}$.

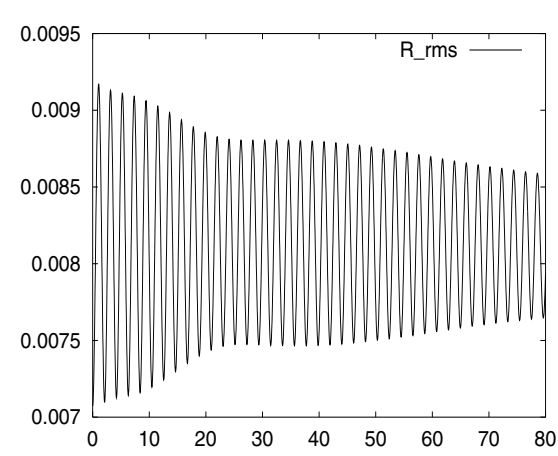

(1)

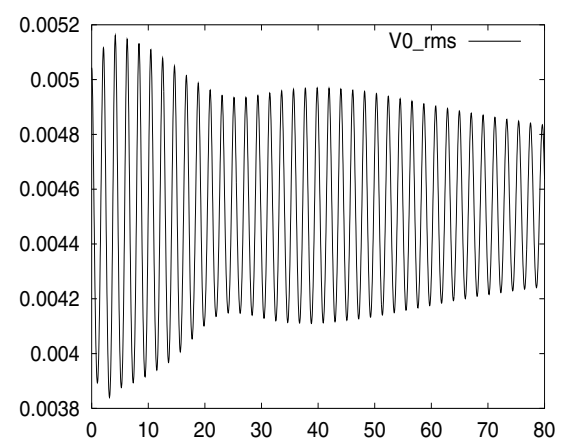

(3)

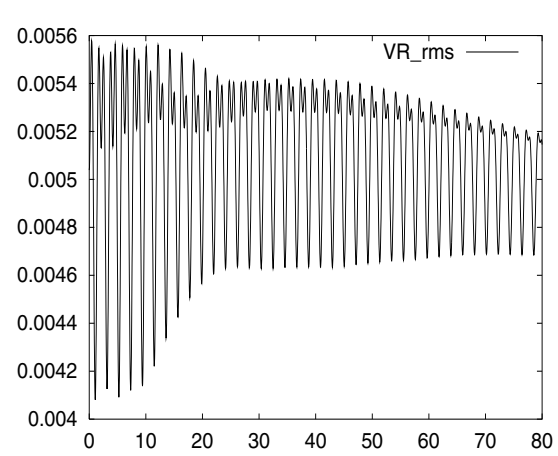

(2)

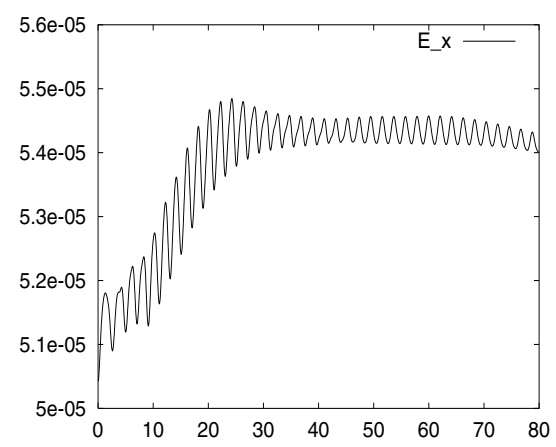

(4)

Fig. 2. (1) $r_{r m s}$, (2) $v_{r_{r m s}}$, (3) $v_{\theta_{r m s}}$ (4) $\epsilon_{x}$ for an axisymmetric Maxwell-Boltzmann beam.

\section{Conclusion}

In this paper, we propose a new axisymmetric solver for the Vlasov equation. The formulation using invariants allows us to do staighforward and efficient parallel 


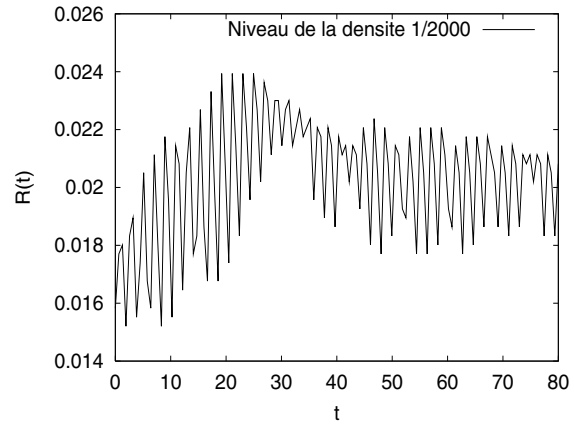

(1)

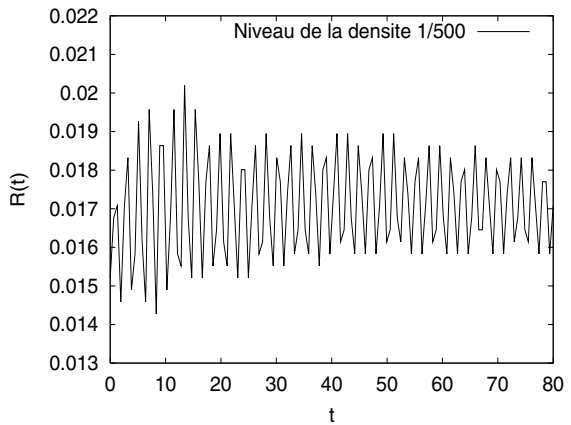

(2)

Fig. 3. Axisymmetric Maxwell-Boltzmann beam. Snapshots of the isolines corresponding to: (1) one two thousandth of the total density, (2) one five hundredth of the total density.
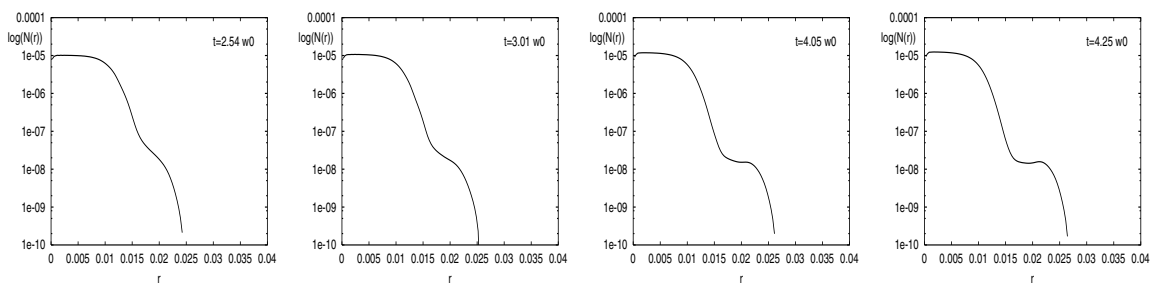

Fig. 4. Axisymmetric Maxwell-Boltzmann beam. Snapshots of slice of density on a logarithmic scale. 
computations. The accuracy of the scheme is such that it is possible to observe halo formations and to get a good description of the distribution function in the phase space. These results are promising to treat more complex problems in accelerator physics.

Acknowledgement: The authors wish to thank Christian Quine for the help he provided with the numerical computations.

\section{References}

1. A. Friedman, D.P Grote, I. Haber, Phys. Fluids B 4 (1992), 2203-2210.

2. P.M. Lapostolle, IEEE Trans. Nucl. Sci. NS 18 (1971), 1101.

3. G. Manfredi, M. Shoucri, M.R. Feix, P. Bertrand, E. Fijalkow, A. Ghizzo, The numerical integration of the Vlasov equation possessing an invariant. J. Comput. Phys. 121 (1995), 298-313.

4. M. Reiser, Theory and design of charged particle beams. Wiley and sons (1994).

5. E. Sonnendrücker, J. Roche, P. Bertrand, A. Ghizzo, The Semi-Lagrangian Method for the Numerical Resolution of Vlasov Equations. J. Comput. Phys. 149: (1998) 201-220.

6. E. Sonnendrücker, J.J. Barnard, A. Friedman, D.P. Grote, S.M. Lund, Simulation of heavy ion beams with a semi-Lagrangian Vlasov solver, Nuclear Instruments and Methods in Physics Research, Section A, 464 (1-3), (2001), 653-661.

7. T.P. Wangler, R.W. Garnett, E.R. Gray, R.D. Ryne, T.S. Wang, Dynamics of beam halo in mismatched beams. XVIII International Linac Conference, Genève, Suisse (1996). 